\title{
A note on a new class of generalized Pearson distribution arising from Michaelis-Menten function of enzyme kinetics
}

\author{
Mohammad Shakil $^{1 *}$, Jai Narain Singh ${ }^{2}$ \\ ${ }^{1}$ Department of Mathematics, Miami Dade College, Hialeah Campus, Hialeah, FL 33012, USA \\ ${ }^{2}$ Department of Mathematics and Computer Science, Barry University, Miami Shores, FL 33161, USA \\ *Corresponding author's E-mail: mshakil@mdc.edu
}

Copyright $\odot 2015$ Mohammad Shakil, Jai Narain Singh. This is an open access article distributed under the Creative Commons Attribution License, which permits unrestricted use, distribution, and reproduction in any medium, provided the original work is properly cited.

\begin{abstract}
Many problems of enzyme kinetics can be described by a function known as the Michaelis-Menten (M-M) function. In this paper, motivated by the importance of Michaelis-Menten function in biochemistry and other biological phenomena, we have introduced a new class of generalized Pearson distribution arising from Michaelis-Menten function. Various properties of this distribution are derived, for example, its probability density function (pdf), cumulative distribution function (cdf), moment, entropy function, and relationships with some well-known continuous probability distributions. The graphs of the pdf and cdf of our new distribution are provided for some selected values of the parameters. It is observed that our new distribution is positively skewed and unimodal. We hope that the findings of this paper will be useful in many applied research problems.
\end{abstract}

2000 Mathematics Subject Classification: 60E05, 62E10, $62 E 15$.

Keywords: Enzyme Kinetics; Generalized Pearson Differential Equation; Generalized Pearson System of Probability Distributions; MichaelisMenten Function.

\section{Introduction}

The Michaelis-Menten function is one of the most important mathematical functions to model many problems of biochemistry and other biological phenomena to describe enzymatic reactions, see, for example, Michaelis and Menten [13], Briggs and Haldane [3], Cleland [5], Fontes et.al. [8], [9], among others. It is defined by the following equation:

$v=f([S])=\frac{V_{\max }[S]}{K_{m}+[S]}$,

where $v$ is the initial velocity in an enzyme-catalyzed reaction, $V_{\max }$ is the maximal velocity, i.e. the velocity attended at very high concentration of substrate $[\mathrm{S}], K_{m}$ is the Michaelis constant and corresponds to the concentration of substrate at which $v=V_{\max } / 2$.

As pointed out by, Fontes et.al. [9], the Michaelis-Menten equation (1) can be reduced to the following forms known as Type I, Type II and Type III respectively:

Type 1: $y=\frac{a x}{b+x}$,

Type 2: $y=\frac{n_{0}+n_{1} x}{d_{0}+d_{1} x}$,

Type 3: $y=h+\frac{a x}{b+x}$, 
where the constants have their usual meanings. For detailed mathematical analysis and applications to enzymatic reactions of the above three types of the Michaelis-Menten equation, see Fontes et.al. [9], among others.

In this paper, motivated by the importance of Michaelis-Menten function in biochemistry and other biological phenomena, we have introduced a new class of generalized Pearson distribution arising from Michaelis-Menten function. The organization of this paper is as follows. Section 2 contains a review of existing classes of generalized Pearson continuous probability distributions as considered by various researchers. We have identified as many as 14 such distributions. Section 3 contains the derivations of the probability density function ( $p d f$ ) and cumulative distribution function (cdf) of our proposed new class of generalized Pearson distribution arising from Michaelis-Menten function. In Section 4, some distributional properties of our proposed new class of generalized Pearson distribution, along with the graphs of the pdf and cdf for some selected values of the parameters, are provided. Section 5 contains some concluding remarks.

\section{Review on existing classes of generalized Pearson system of distributions}

A continuous probability distribution belongs to the Pearson system if, for a positive continuous random variable $X$, its probability density function $(p d f) f$ satisfies a differential equation of the form

$\frac{1}{f(x)} \frac{d f_{X}(x)}{d x}=-\frac{x+a}{b x^{2}+c x+d}$,

where $a, b, c$, and $d$ are real parameters such that $f$ is a $p d f$. The shapes of the $p d f$ depend on the values of these parameters, based on which Pearson [16, 17] classified these distributions into a number of types known as Pearson Types I - VI. Later, in another paper, Pearson [18] defined more special cases and subtypes known as Pearson Types VII - XII. Many well-known distributions belong to these types of Pearson distributions which include Normal and Student's $t$ distributions (Pearson Type VII), Beta distribution (Pearson Type I), Gamma distribution (Pearson Type III), among others. For details on the Pearson systems of continuous probability distributions, the interested readers are referred to Johnson et. al. [12]. In recent years, many researchers have considered a generalization of the Pearson system, known as generalized Pearson system of differential equation (GPE), given by

$\frac{1}{f(x)} \frac{d f_{X}(x)}{d x}=\frac{\sum_{j=0}^{m} a_{j} x^{j}}{\sum_{j=0}^{n} b_{j} x^{j}}$,

where $m, n \in \mathrm{N} /\{0\}$ and the coefficients $a_{j}$ and $b_{j}$ are real parameters. The system of continuous univariate $p d f$ ' $s$ generated by GPE is called a generalized Pearson system which includes a vast majority of continuous $p d f$ ' $s$ by proper choices of these parameters. We have identified as many as 14 such distributions, which are provided below:

i) Roy [21] studied GPE, when $m=2, n=3, b_{0}=0$, to derive five frequency curves whose parameters depend on the first seven population moments.

ii) Dunning and Hanson [7] used GPE in his paper on generalized Pearson distributions and nonlinear programming.

iii) Cobb et. al. [6] extended Pearson's class of distributions to generate multimodal distributions by taking the polynomial in the numerator of GPE of degree higher than one and the denominator, say, $v(x)$, having one of the following forms:

a) $v(x)=1, \quad-\infty<x<\infty$,

b) $v(x)=x, \quad 0<x<\infty$,

c) $v(x)=x^{2}, \quad 0<x<\infty$,

d) $\quad v(x)=x(1-x), \quad 0<x<1$.

iv) Chaudhry and Ahmad [4] studied another class of generalized Pearson distributions when $m=4, n=3, b_{0}=b_{1}=b_{2}=0, \frac{a_{4}}{2 b_{3}}=-2 \alpha, \frac{a_{0}}{2 b_{3}}=2 \beta, b_{3} \neq 0$.

v) Lefevre et al. [13] studied characterization problems based on some generalized Pearson distributions.

vi) Considering the following class of GPE $\frac{1}{f(x)} \frac{d f_{X}(x)}{d x}=\frac{a_{0}+a_{1} x+a_{2} x^{2}}{b_{0}+b_{1} x+b_{2} x^{2}}$,

Sankaran [22] proposed a new class of probability distributions and established some characterization results based on a relationship between the failure rate and the conditional moments.

vii) Stavroyiannis and Stavroulakis [28] studied generalized Pearson distributions in the context of the superstatistics with non-linear forces and various distributions. 
viii) Rossani and Scarfone [20] have studied GPE in the following form $\frac{1}{f(x)} \frac{d f_{X}(x)}{d x}=-\frac{a_{0}+a_{1} x+a_{2} x^{2}}{b_{0}+b_{1} x+b_{2} x^{2}}$, and used it to generate generalized Pearson distributions in order to study charged particles interacting with an electric and/or a magnetic field.

ix) Shakil et. al. [24] defined a new class of generalized Pearson distributions based on the following differential equation $\frac{d f_{X}(x)}{d x}=\left(\frac{a_{0}+a_{1} x+a_{2} x^{2}}{b_{1} x}\right) f_{X}(x), \quad b_{1} \neq 0$,

which is a special case of the GPE (2), when $m=2, n=1$, and $b_{0}=0$. The solution to the differential equation (3) is

given by $f_{X}(x)=C x^{\alpha} \exp \left(-\mu x^{2}-\beta x\right), \quad \alpha>0, \beta>0, \mu>0, x>0$,

where $\mu=-\frac{a_{2}}{2 b_{1}}, \alpha=\frac{a_{0}}{b_{1}}, \beta=-\frac{a_{1}}{b_{1}}, b_{1} \neq 0$, and $C$ is the normalizing constant given by

$C=\frac{(2 \mu)^{(\alpha+1) / 2}}{\Gamma(\alpha+1) \exp \left(\beta^{2} /(8 \mu)\right) D_{-(\alpha+1)}(\beta /(\sqrt{2 \mu}))}$,

where $D_{p}(z)$ denotes the well-known parabolic cylinder function.

x) Shakil and Kibria [23] consider the GPE (2) in the following form

$\frac{d f_{X}(x)}{d x}=\left(\frac{a_{0}+a_{p} x^{p}}{b_{1} x+b_{p+1} x^{p+1}}\right) f_{X}(x), \quad b_{1} \neq 0, b_{p+1} \neq 0, x>0$,

when $m=p, n=p+1, a_{1}=a_{2}=\cdots=a_{p-1}=0$, and $b_{0}=b_{2}=\cdots=b_{p}=0$. The solution to the differential equation (6)

is given by $f_{X}(x)=C x^{\mu-1}\left(\alpha+\beta x^{p}\right)^{-v}, x>0, \alpha>0, \beta>0, \mu>0, v>0$, and $p>0$,

where $\alpha=b_{1}, \beta=b_{p+1}, \mu=\frac{a_{0}+b_{1}}{b_{1}}, v=\frac{a_{0} b_{p+1}-a_{p} b_{1}}{p b_{1} b_{p+1}}, b_{1} \neq 0, b_{p+1} \neq 0$, and $C$ is the normalizing constant given

by $C=\frac{p(\alpha)^{v-\frac{\mu}{p}}(\beta)^{\frac{\mu}{p}}}{\mathrm{~B}\left(\frac{\mu}{p}, v-\frac{\mu}{p}\right)}$,

where $\mathrm{B}\left(\frac{\mu}{p}, v-\frac{\mu}{p}\right)$ denotes the well-known beta function, and $v>\frac{\mu}{p}$.

xi) Shakil et. al. [25] consider the GPE (2) in the following form

$\frac{d f_{X}(x)}{d x}=\left(\frac{a_{0}+a_{p} x^{p}+a_{2 p} x^{2 p}}{b_{p+1} x^{p+1}}\right) f_{X}(x), \quad b_{p+1} \neq 0, x>0$,

where $m=2 p, n=p+1, a_{1}=a_{2}=\cdots=a_{p-1}=a_{p+1}=\cdots=a_{2 p-1}=0$, and $b_{0}=b_{1}=b_{2}=\cdots=b_{p}=0$. The solution to the differential equation (9) is given by

$f_{X}(x)=C x^{v-1} \exp \left(-\alpha x^{p}-\beta x^{-p}\right), x>0, \alpha \geq 0, \beta \geq 0,-\infty<v<\infty$,

where $\alpha=-\frac{a_{2 p}}{p b_{p+1}}, \beta=\frac{a_{0}}{p b_{p+1}}, v=\frac{a_{p}+b_{p+1}}{b_{p+1}}, b_{p+1} \neq 0, p>0$, and $C$ is the normalizing constant given by

$C=\frac{p}{2}\left(\frac{\alpha}{\beta}\right)^{\frac{v}{2 p}} \frac{1}{K_{\frac{v}{p}}(2 \sqrt{\alpha \beta})}$,

where $K_{\frac{v}{p}}(2 \sqrt{\alpha \beta})$ denotes the well-known modified Bessel function of third kind. For the characterizations

of the above continuous probability distribution, due to Shakil et. al. [25], known in the literature as the Shakil-KibriaSingh (SKS) distribution, the interested readers are referred to Hamedani [11] where the the Shakil-Kibria-Singh (SKS) distribution has been characterized by Hamedani [11] based on a simple relationship between two truncated moments, and the hazard function.

xii) Hamedani [11] has defined a new variation of the continuous probability distribution (10) in a bounded domain. The $p d f$ of Hamedani's distribution is given by 
$f(x)=C p x^{-(p+1)}\left(\beta-\alpha x^{2 p}\right) \exp \left(-\alpha x^{p}-\beta x^{-p}\right), \quad 0<x<\left(\frac{\beta}{\alpha}\right)^{\frac{1}{2 p}}$,

where $\alpha>0, \beta>0$, and $p>0$ are parameters and $C=\exp \{2 \sqrt{\alpha \beta}\}$ is the normalizing constant.

The $c d f$ corresponding to the $p d f$ (12) is given by

$F(x)=C \exp \left(-\alpha x^{p}-\beta x^{-p}\right), \quad 0<x<\left(\frac{\beta}{\alpha}\right)^{\frac{1}{2 p}}$.

For the special case of $\alpha=\beta$, we have

$f(x)=\alpha p e^{2 \alpha} x^{-(p+1)}\left(1-x^{2 p}\right) \exp \left(-\alpha\left(x^{p}+x^{-p}\right)\right), 0<x<\left(\frac{\beta}{\alpha}\right)^{\frac{1}{2 p}}$,

where $\alpha>0$ and $p>0$ are parameters. As pointed out by Hamedani [11], the $p d f f$ given by (12) satisfies the following differential equation $\frac{1}{f(x)} \frac{d f(x)}{d x}=\frac{\beta^{2} p-\beta(p+1) x^{p}-2 \alpha \beta p x^{2 p}-\alpha(p-1) x^{3 p}+\alpha^{2} p x^{4 p}}{\beta x^{p+1}-\alpha x^{3 p+1}}$,

which is a special case of GPE (2). For the characterization of the $p d f$ in Eq. (12), when $p \in \mathrm{N} /\{0\}$, the interested readers are referred to Hamedani [11].

xiii) Ahsanullah et. al. [2] defined a new class of distributions as solutions of the GPE (2). They considered the following differential equation

$\frac{d f_{X}(x)}{d x}=\left(\frac{a_{1}+a_{2} x+a_{3} x^{2}}{b_{3} x^{2}+b_{4} x^{3}}\right) f_{X}(x)$,

which is a special case of the generalized Pearson Eq. (2) when $m=2, n=3$. Putting $b_{3}=1, b_{4}=\gamma, a_{1}=\beta \gamma$,

$\mathrm{a}_{2}=\beta-\gamma+\gamma v, \mathrm{a}_{3}=v+\mu-2, \mathrm{x}>0$, in (3), we have

$\frac{1}{f_{X}(x)} \frac{d f_{X}(x)}{d x}=\frac{\beta \gamma+(\beta-\gamma+\gamma v) x+(\mu+v-2) x^{2}}{x^{3}+\gamma x^{2}}=\frac{v-1}{x}+\frac{\mu-1}{x+\gamma}+\frac{\beta}{x^{2}}$,

where we assume that $\beta>0, \gamma>0,0<v<1,0<\mu<1,1-\mu>v>0$.

Integrating the above equation, we have

$f_{X}(x)=C x^{\nu-1}(x+\gamma)^{(\mu-1)} \exp \left(-\beta x^{-1}\right), \quad 0<x<\infty$,

Using the equation (3.471.7), Page 340 of Gradshteyn and Ryzhik [10], we easily obtain the following normalizing constant as

$\frac{1}{C}=\beta^{(\nu-1) / 2} \gamma^{\frac{v-1}{2}+\mu} \Gamma(1-\mu-v) \exp \left(\frac{\beta}{2 \gamma}\right) W_{\frac{v-1}{2}+\mu, \frac{-v}{2}}\left(\frac{\beta}{\gamma}\right)$,

where $W_{a, b}(z)$ denotes the well-known Whittaker function (see Abramowitz and Stegun [1], page 505, chapter 13).

xiv) Recently, Stavroyiannis [27] defined a new class of distributions as solutions of the GPE (2) by considering the following differential equation

$\frac{1}{f(x)} \frac{d f_{X}(x)}{d x}=\frac{\sum_{j=0}^{5} a_{j} x^{j}}{\sum_{j=0}^{6} b_{j} x^{j}}$

which is a special case of the generalized Pearson Eq. (2) when $m=5, n=6$. By taking special values of the coefficients $a_{j}$ and $b_{j}$, Stavroyiannis [27] obtained the GPE in the following form

$\frac{1}{f(x)} \frac{d f(x)}{d x}=-\frac{a^{5} v+2 a^{4} m(x-\lambda)+a^{3} v(x-\lambda)^{2}+2 a^{2}(1+2 b) m(x-\lambda)^{3}+a b v(x-\lambda)^{4}+4 b m(x-\lambda)^{5}}{\left(a^{2}+(x-\lambda)^{2}\right)\left(a^{4}+a^{2}(x-\lambda)^{2}+b(x-\lambda)^{4}\right)}$

with its solution given by the following probability density function:

$f(x)=C \frac{1}{\left(1+\left(\frac{x-\lambda}{a}\right)^{2}+b\left(\frac{x-\lambda}{a}\right)^{4}\right)^{m}} \exp v\left(-\tan ^{-1}\left(\frac{x-\lambda}{a}\right)\right)$

where $\lambda$ is the location parameter, $a>0$ is the scale parameter, $m>\frac{1}{2}$ and $b \geq 0$ control the kurtosis, $v$ is the asymmetry parameter, and $C$ is the normalization constant. As pointed by Stavroyiannis [27], the above distribution 
with the $p d f$ (19) includes an extra fourth order term in the denominator to account for fat and thick-tails for the case of $b>0$. The distribution becomes double peaked for the case of a negative $b$ coefficient, while for $b=0$ the PearsonIV distribution is regained. For details on these, the interested readers are referred to Stavroyiannis [27].

\section{A new class of generalized pearson distribution arising from michaelis- menten function}

In this section, for a positive continuous random variable $\mathrm{X}$, we define a new class of generalized Pearson distributions based on the following differential equation

$\frac{1}{f(x)} \frac{d f(x)}{d x}=-\frac{a_{1}+b_{1} x}{a_{2}+b_{2} x}$,

$m=1, n=1, a_{2}=a_{3}=\cdots=a_{m}=0, b_{2}=b_{3}=\cdots=b_{n}=0$, and where the expression, $\frac{a_{1}+b_{1} x}{a_{2}+b_{2} x}$, i.e., the ratio of two

polynomials of first degree in $x$, with $a_{1}, b_{1} \geq 0, a_{2}, b_{2}>0, a_{1} b_{2} \neq a_{2} b_{1}$ (except when $a_{1}, b_{1}=0$ ), is known as MichaelisMenten function. The solution to the differential equation (20) is easily obtained as follows

$f_{X}(x)=C(1+\alpha x)^{\mu}(\gamma+\delta x)^{-\theta} e^{-\lambda x}, \alpha, \gamma, \delta>0, \mu, \theta, \lambda \geq 0, x>0$,

where $\mu=\frac{a_{2} b_{1}}{\left(b_{2}\right)^{2}}, \alpha=\frac{b_{2}}{a_{2}}, \gamma=a_{2}, \delta=b_{2}, \theta=\frac{a_{1}}{b_{2}}, \lambda=\frac{b_{1}}{b_{2}},\left(a_{2}, b_{2} \neq 0\right)$, and $C$ denotes the normalizing constant.

\subsection{Expressions for the normalizing constant}

In order that the right side of the Eq. (21) represents a probability density function (pdf), we must have

$\int_{0}^{\infty} f_{X}(x) d x=\int_{0}^{\infty} C(1+\alpha x)^{\mu}(\gamma+\delta x)^{-\theta} e^{-\lambda x} d x=1$.

i) In Eq. (22), using twice the binomial series representation $(1+w)^{-s}=\sum_{k=0}^{\infty} \frac{(s)_{k}(-w)^{k}}{k !}$, for any real value of $s$, and Eq. 3.351.3/ P. 310 of Gradshteyn and Ryzhik [10], where $(s)_{k}=\frac{\Gamma(s+k)}{\Gamma(s)}=s(s+1) \ldots(s+k-1),(s \neq 0)$, and $(s)_{0}=1$, denote the Pochhammer symbol, the normalizing constant $C$ is easily given by

$C=\left[\sum_{k=0}^{\infty} \sum_{j=0}^{\infty} \frac{(-1)^{k}(\theta)_{k}(\mu)_{j} \gamma^{-\theta}\left(\frac{\delta}{\gamma}\right)^{k} \alpha^{j} \lambda^{-k-j-1}(k+j) !}{k ! j !}\right]^{-1}$

ii) Again, in Eq. (22), using the binomial series representation $(1+w)^{-s}=\sum_{k=0}^{\infty} \frac{(s)_{k}(-w)^{k}}{k !}$, for any real value of $s$, and Equation 2.3.6.9 of Prudnikov et al., Vol. 1 [19], the expression for the normalizing constant $C$ is easily obtained, after simplification, as follows

$C=\left[\sum_{j=0}^{\infty} \delta^{-\theta} \alpha^{j}(\mu)_{j}\left(\frac{\gamma}{\delta}\right)^{j+1-\theta} \Psi\left(j+1, j+2-\theta ; \frac{\lambda \gamma}{\delta}\right)\right]^{-1}$

where $\Psi(p, q ; z)=\frac{1}{\Gamma(p)} \int_{0}^{\infty} e^{-z t} t^{p-1}(1+p)^{q-p-1} d t$ is known as Kummer's (or degenerate hypergeometric) function of the second kind, see, for example, Abramowitz and Stegun [1], Gradshteyn and Ryzhik [10], and Oldham et. al. [15], among others. 


\subsection{Expression for the cumulative distribution function}

Using twice the binomial series representation $(1+w)^{-s}=\sum_{k=0}^{\infty} \frac{(s)_{k}(-w)^{k}}{k !}$, for any real value of $s$, and Eq. 3.381.1/P. 317 of Gradshteyn and Ryzhik [10], the cumulative distribution function (cdf ) of our new distribution is easily obtained as follows

$F_{X}(x)=\int_{0}^{x} f_{X}(x) d x=C \sum_{k=0}^{\infty} \sum_{j=0}^{\infty} \frac{(-1)^{k}(\theta)_{k}(\mu)_{j} \gamma^{-\theta}\left(\frac{\delta}{\gamma}\right)^{k} \alpha^{j} \lambda^{-k-j-1} \gamma(k+j+1, \lambda x)}{k ! j !}$

where $\gamma(s, z)=\int_{0}^{z} t^{s-1} e^{-t} d t$ denotes the incomplete gamma function, and $C$ denote the normalizing constant given by the equation (23).

\section{Distributional properties}

In what follows, some properties of our proposed distribution are given below.

\subsection{Graphs of the PDF and CDF}

The possible shapes of the $p d f f(x)$ in Eq. (21) and the $c d f F(x)$ in Eq. (23) are provided for some selected values of the parameters in the following Figures 1-6. The effects of parameters can be easily seen from these graphs. Also, it is clear from these graphs that our proposed distributions of the random variable $X$ are positively (that is, right) skewed and unimodal.
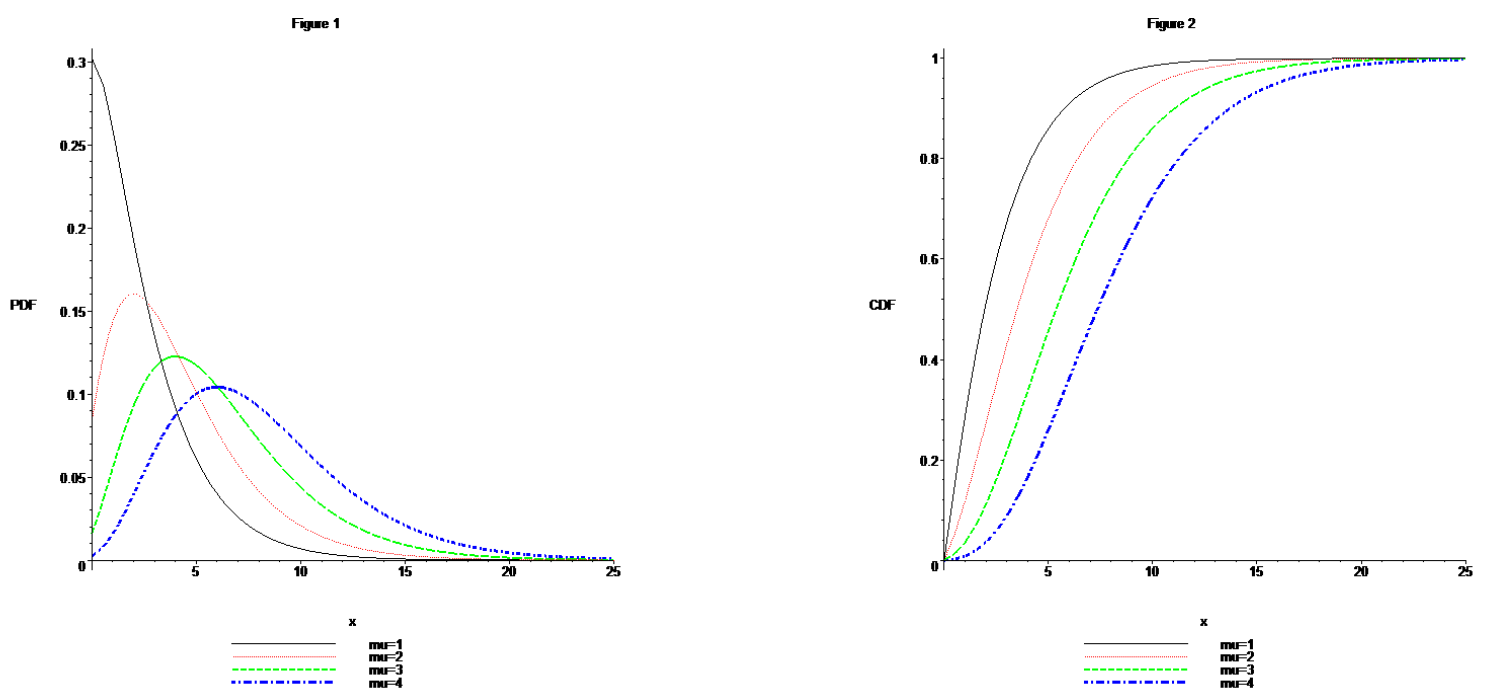

Fig. 1: PDF and Figure 2: CDF For $\mu=1,2,3,4$ when $\alpha=1, \gamma=1, \delta=1, \theta=0.5, \lambda=0.5$. 

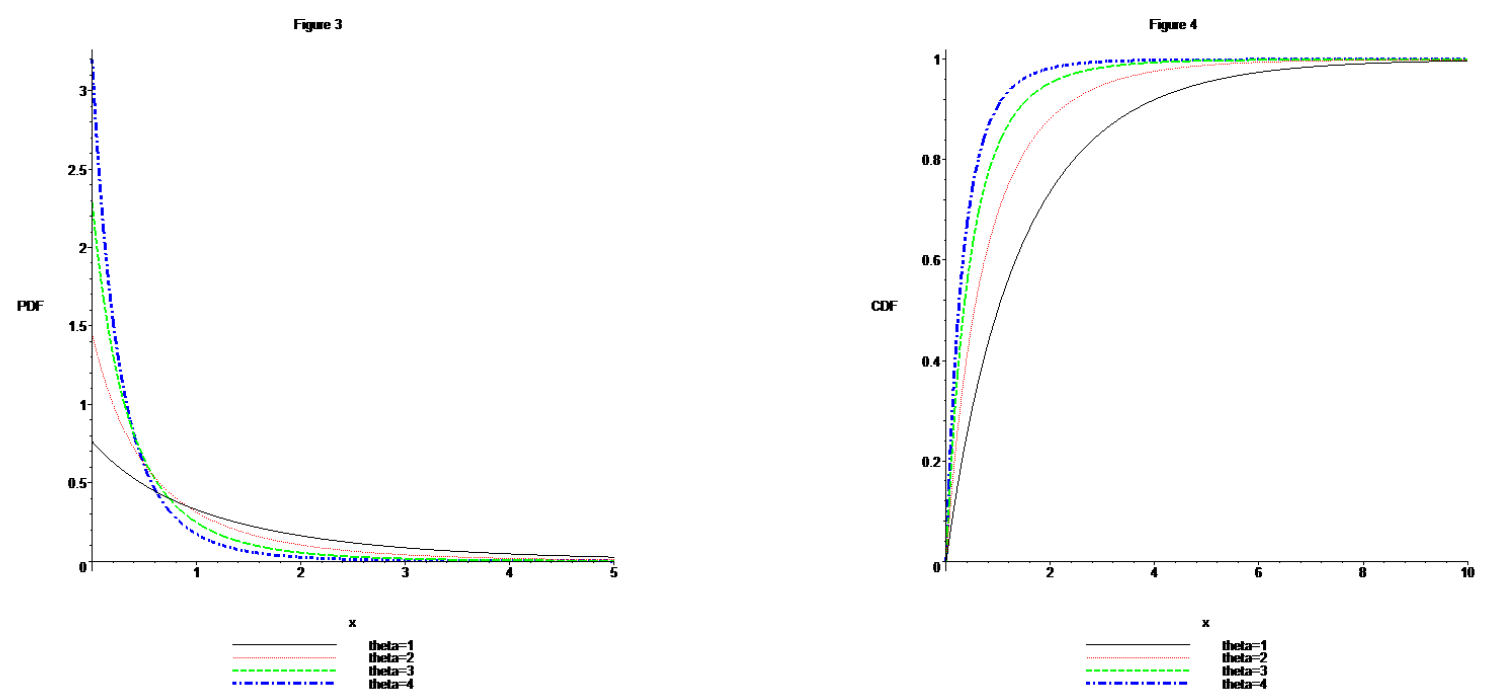

Fig. 3: PDF and Fig. 4: CDF For $\theta=1,2,3,4$ when $\alpha=1, \gamma=1, \delta=1, \mu=0.5, \lambda=0.5$.
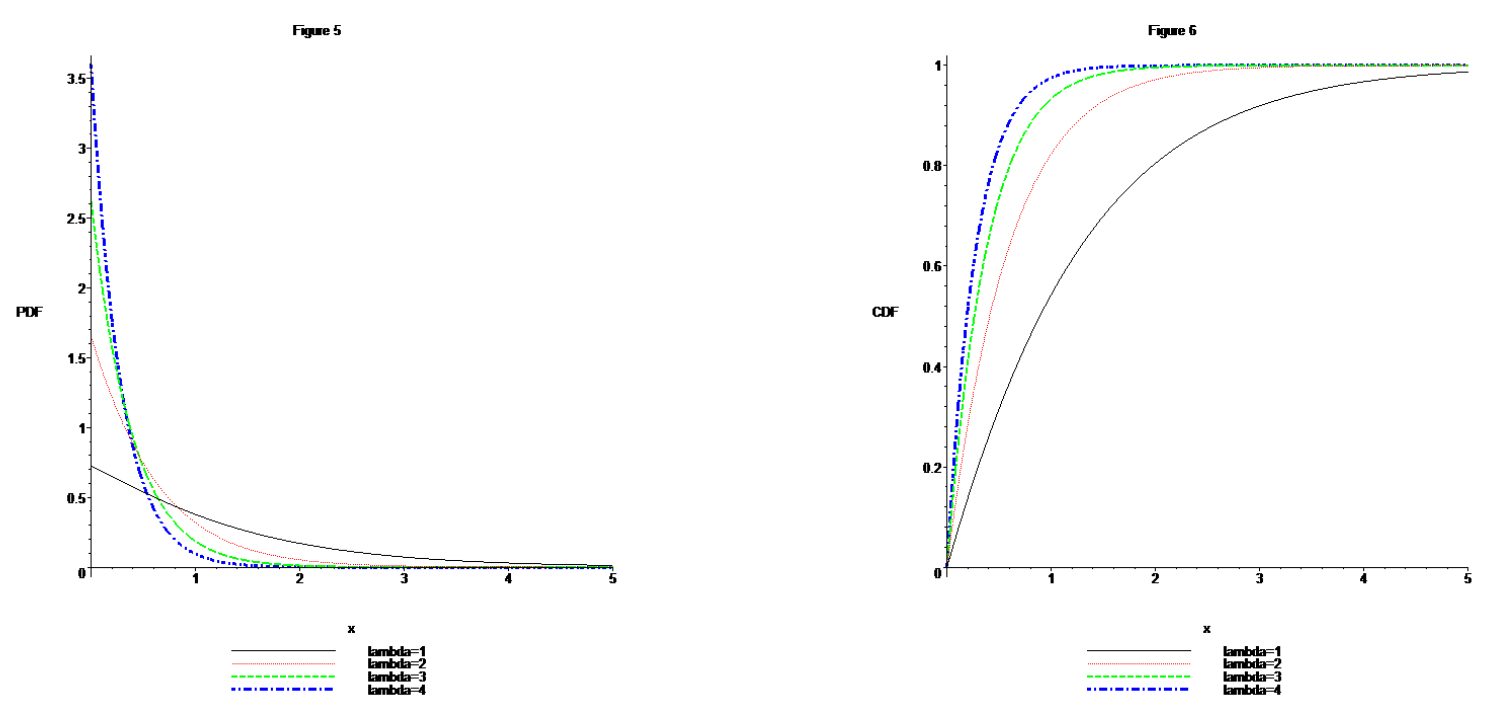

Fig. 5: PDF and Fig. 6: CDF for $\lambda=1,2,3,4$ when $\alpha=1, \gamma=1, \delta=1, \mu=1, \theta=0.5$.

\section{2. nth Moment}

In what follows, we derive the moments of our proposed distribution. We have $E\left(X^{n}\right)=\int_{0}^{\infty} x^{n} f_{X}(x) d x=C \int_{0}^{\infty} x^{n}(1+\alpha x)^{\mu}(\gamma+\delta x)^{-\theta} e^{-\lambda x} d x$.

In Eq. (26), using the binomial series representation $(1+w)^{-s}=\sum_{k=0}^{\infty} \frac{(s)_{k}(-w)^{k}}{k !}$, for any real value of $s$, and Eq. 2.3.6.9 of Prudnikov et. al., Vol. 1 [19], the following expression for the $n t h$ moment is easily obtained:

$E\left(X^{n}\right)=C \sum_{j=0}^{\infty} \frac{\delta^{-\theta} \alpha^{j}(\mu)_{j}\left(\frac{\delta}{\gamma}\right)^{n+j+1-\theta} \Gamma(n+j+1) \Psi\left(n+j+1, n+j+2-\theta ; \frac{\lambda \delta}{\gamma}\right)}{j !}$,

where $C$ denotes the normalizing constant given by (24), $\Psi(p, q ; z)=\frac{1}{\Gamma(p)} \int_{0}^{\infty} e^{-z t} t^{p-1}(1+p)^{q-p-1} d t$ is known as Kummer's (or degenerate hypergeometric) function of the second kind, and $\Gamma$ (.) denotes the gamma function defined by $\Gamma(s)=\int_{0}^{\infty} t^{s-1} e^{-t} d t$, see, for example, Abramowitz and Stegun [1], Gradshteyn and Ryzhik [10], and Oldham et. al. 
[15], among others. Taking $n=1,2,3, \ldots$, in Eq. (27), we can easily obtain the moments of different orders, including the variance, $\sigma^{2}$, of our proposed distribution which can be obtained by using the formula: $\sigma^{2}=E\left(X^{2}\right)-(E(X))^{2}$.

\subsection{Shannon entropy}

An entropy provides an excellent tool to quantify the amount of information (or uncertainty) contained in a random observation regarding its parent distribution (population). A large value of entropy implies the greater uncertainty in the data. As proposed by Shannon [26], if $X$ is a none-negative continuous random variable with pdf $f_{X}(x)$, then Shannon's entropy of $X$, denoted by $h[f]$ or $h(X)$, is defined as

$$
h[f]=h(X)=E\left[-\ln \left\{f_{X}(x)\right\}\right]=-\int_{0}^{\infty} f_{X}(x) \ln \left\{f_{X}(x)\right\} d x .
$$

Now, in Eq. (28) Above, using the pdf $f(x)$ of the our proposed distribution, as given in Eq. (21), and then integrating and simplifying, Shannon entropy of our proposed distribution is easily obtained as follows

$$
h[f]=h(X)=-\ln C+\theta \ln \lambda-\lambda E(X)-\mu \sum_{i=1}^{\infty} \frac{(-1)^{i} \alpha^{i} E\left(X^{i}\right)}{i}+\theta \sum_{j=1}^{\infty} \frac{(-1)^{j}\left(\frac{\delta}{\gamma}\right)^{j} E\left(X^{j}\right)}{j},
$$

where $C$ denotes the normalizing constant given by Eq. (24), and $E(X), E\left(X^{i}\right)$ and $E\left(X^{j}\right)$ denote the first, ith and $j$ th moments respectively of our proposed distribution, and can be obtained by taking $n=1, i$ and $j$ in Eq. (27) respectively.

\subsection{Distributional relationships}

It is easy to see that, by a simple transformation of the variable $x$ or by taking special values of the parameters $\{\alpha, \gamma, \delta>0 ; \mu, \theta, \lambda \geq 0\}$, number distributions are special cases of our proposed distribution as stated below.

i) Pearson III Distribution (when $\theta=0$ ): For the sake of motivation, the derivation of Pearson III distribution (when $\theta=0$ ) from our proposed distribution with PDF as given in (21) is provided below. Thus, when $\theta=0$, we have $f_{X}(x)=C(\alpha+\beta x)^{\mu} e^{-\lambda x}, \alpha>0, \beta>0, \mu>0, x>0, \lambda>0$,

where $\mu=-\frac{a_{2}}{2 b_{1}}, \alpha=\frac{a_{0}}{b_{1}}, \beta=-\frac{a_{1}}{b_{1}}, b_{1} \neq 0$, and $C$ is the normalizing constant given by $C=\beta^{-\mu} \lambda^{\mu+1} e^{-\left(\frac{\alpha \lambda}{\beta}\right)}\left[\Gamma\left(\mu+1, \frac{\alpha \lambda}{\beta}\right)\right]^{-1}$,

where $\Gamma(s, z)=\int_{z}^{\infty} t^{s-1} e^{-t} d t$ denotes the incomplete gamma function.

The CDF of Pearson III distribution (when $\theta=0$ ) is given by $F(x)=\frac{C}{\lambda}\left(\frac{\beta}{\lambda}\right)^{\mu} e^{\frac{\alpha \lambda}{\beta}}\left[\gamma\left(\mu+1, \frac{\alpha \lambda}{\beta}+\lambda x\right)-\gamma\left(\mu+1, \frac{\alpha \lambda}{\beta}\right)\right]$,

where $\gamma(s, z)=\int_{0}^{z} t^{s-1} e^{-t} d t$ denotes the incomplete gamma function, and $C$ denote the normalizing constant as given above. The first, second, and nth moments of Pearson III distribution are obtained as follows:

First Moment: $E(X)=C \beta^{\mu} \sum_{m=0}^{\infty}\left(\begin{array}{l}\mu \\ m\end{array}\right)\left(\frac{\alpha}{\beta}\right)^{m} \frac{1}{\lambda^{\mu-m+2}} \Gamma(\mu-m+2)$,

Second Moment: $E\left(X^{2}\right)=C \beta^{\mu} \sum_{m=0}^{\infty}\left(\begin{array}{l}\mu \\ m\end{array}\right)\left(\frac{\alpha}{\beta}\right)^{m} \frac{1}{\lambda^{\mu-m+3}} \Gamma(\mu-m+3)$,

nth Moment: $E\left(X^{n}\right)=C \beta^{\mu} \sum_{m=0}^{\infty}\left(\begin{array}{l}\mu \\ m\end{array}\right)\left(\frac{\alpha}{\beta}\right)^{m} \frac{1}{\lambda^{\mu-m+n}} \Gamma(\mu-m+n)$, 
where $\Gamma(s)=\int_{0}^{\infty} t^{s-1} e^{-t} d t$ denotes the gamma function.

Entropy: $h[f]=h(X)=-\ln C-\mu \ln \alpha+\lambda E(X)+\sum_{j=1}^{\infty} \frac{(-1)^{j} \mu\left(\frac{\beta}{\alpha}\right)^{j}}{j} E\left(X^{j}\right)$,

where $E(X)$ and $E\left(X^{j}\right)$ denote the first and the $j$ th moments of Pearson III distribution.

ii) Pearson VIII Distribution (when $\mu=0, \lambda=0$ ).

iii) Pearson IX Distribution (when $\lambda=0, \theta=0$ ).

iv) Pearson X Distribution (when $\mu=0, \theta=0$ ).

v) A Special Case of Our Proposed Distribution (when $\mu=0$ ): Thus, when $\mu=0$ in our proposed distribution with PDF as given in (21), we have

$f_{X}(x)=C(\gamma+\delta x)^{-\theta} e^{-\lambda x}, \alpha, \gamma, \delta>0 ; \theta, \lambda \geq 0 ; x>0$, where $C$ denotes the normalizing constant given by $C=\left(\frac{\gamma}{\delta}\right)^{\theta}\left[\gamma \Psi\left(1,2-\theta ; \frac{\lambda \gamma}{\delta}\right)\right]^{-1}$,

which is easily obtained by using Equation 2.3.6.9 of Prudnikov et al., Vol. 1 [19], where $\Psi(p, q ; z)=\frac{1}{\Gamma(p)} \int_{0}^{\infty} e^{-z t} t^{p-1}(1+p)^{q-p-1} d t$ is known as Kummer's (or degenerate hypergeometric) function of the second kind, see, for example, Abramowitz and Stegun [1], Gradshteyn and Ryzhik [10], and Oldham et. al. [15], among others.

vi) Distribution of the Product of the PDF's of the Exponential and Some Members of the Family of Burr Distributions (Lomax, or Pareto Type I, or Pareto Type II): It is easy to see that, by a simple transformation of the variable $x$ or by taking special values of the parameters $\{\alpha, \gamma, \delta>0 ; \theta, \lambda \geq 0\}$, the pdf of the above special case (v) of our proposed distribution (when $\mu=0$ ) can be expressed as the pdf of the product of the pdf's of the exponential and some members of the family of Burr distributions (such as Lomax, or Pareto Type I, or Pareto Type II distributions).

\section{Concluding remarks}

In this paper, we have introduced a new class of generalized Pearson distribution arising from Michaelis-Menten function. Also, we have reviewed existing classes of continuous probability distributions which can be generated from the generalized Pearson system of differential equation (GPE), as given in Eq. (2). We have identified as many as fourteen such distributions. Various properties of our proposed distribution are derived, for example, its probability density function (pdf), cumulative distribution function (cdf), moment, entropy function, and relationships with some well-known continuous probability distributions. The graphs of the pdf and cdf of our proposed distribution are provided for some selected values of the parameters. It is observed that our proposed distribution is positively skewed and unimodal. We hope that the findings of this paper will be useful in many applied research problems. Some open problems and direction for future research for our proposed generalized Pearson distribution are characterization, estimation of parameters, applications to real world problems, Bayesian analysis, regression analysis, among others. Further, we hope that our proposed attempt will be helpful in designing a new approach of unifying different families of distributions based on the generalized Pearson differential equation. Some other open problems are following:

i) Can we unify all continuous probability distributions (known \& unknown) through GPDE?

ii) Can we prove Existence \& Uniqueness Theorem of Solutions for GPDE?

iii) Can we establish a Fixed Point Theorem for GPDE?

\section{Acknowledgment}

The authors would like to thank the editor for helpful suggestions and review process which improved the quality and presentation of the paper. 


\section{Dedication}

The authors would also like to dedicate this paper to Michaelis and Menten who published their seminal paper [Michaelis, L., and Menten, M. L. (1913). Die Kinetik der Invertinwirkung, Biochem. Z., 49, 333-369] on enzymatic reactions a century ago.

\section{References}

[1] Abramowitz, M., and Stegun, I. A. (1970). Handbook of Mathematical Functions, with Formulas, Graphs, and Mathematical Tables. Dover, New York. Bondesson, L. (1979). A general result on infinite divisibility, Annals of Probability, 7 965-979.

[2] Ahsanullah, M., Shakil, M., and Kibria, B. M. G. (2013). On a probability distribution with fractional moments arising from generalized Pearson system of differential equation and its characterization. International Journal of Advanced Statistics and Probability, 1 (3), 132-141. http://dx.doi.org/10.14419/ijasp.v1i3.1435.

[3] Briggs, G.E., and Haldane, J.B.S. (1925). A note on the kinematics of enzyme action. Biochem J 19 (2): 338-339.

[4] Chaudhry, M. A., and Ahmad, M. (1993). On a probability function useful in size modeling. Canadian Journal of Forest Research, 23(8), 1679-1683. http://dx.doi.org/10.1139/x93-209.

[5] Cleland, W.W. (1963) the kinetics of enzyme-catalyzed reaction with two or more substrates or products. I. Nomenclature and rate equations. Biochem. Biophys. Aca 67, pp. 104-137. http://dx.doi.org/10.1016/0926-6569(63)90211-6.

[6] Cobb, L., Koppstein, P., and Chen, N. H. (1983). Estimation and moment recursion relations for multimodal distributions of the exponential family. Journal of the American Statistical Association, 78(381), 124-130. http://dx.doi.org/10.1080/01621459.1983.10477940.

[7] Dunning, K., and Hanson, J. N. (1977). Generalized Pearson distributions and nonlinear programming. Journal of Statistical Computation and Simulation, Volume 6, Issue 2, 115 - 128. http://dx.doi.org/10.1080/00949657708810176.

[8] Fontes, R., Reberiro, J. M., and Sillero, A. (1999) Inhibition and activation of enzymes. The effect of a modifier on the reaction rate and on kinetic parameters. Acta Biochim. Polon. 47, 233-258.

[9] Fontes, R., Meireles Ribeiro, J., and Sillero, A. (2000). An easy procedure to transform the ratio of two polynomials of first degree into Michaelis-Menten-type equations. Application to the ordered Uni Bi enzyme mechanism. Acta Biochimica Polonica, 47(1), 259-268.

[10] Gradshteyn, I. S. and Ryzhik, I. M. (1980). Table of Integrals, Series, and Products (6th edition). Academic Press, San Diego.

[11] Hamedani, G. G. (2011). Characterizations of the Shakil-Kibria-Singh Distribution. Austrian Journal of Statistics, 40 (3), $201-207$.

[12] Johnson, N. L., Kotz, S., and Balakrishnan, N. (1994). Continuous Univariate Distributions, (volume 1, second edition). John Wiley \& Sons, New York.

[13] Lefevre, C., Papathanasiou, V., and Utev, S. (2002). Generalized Pearson distributions and related characterization problems. Annals of the Institute of Statistical Mathematics, 54, 731-742. http://dx.doi.org/10.1023/A:1022403132741.

[14] Michaelis, L., and Menten, M.L. (1913). "Die Kinetik der Invertinwirkung". Biochem Z 49: 333-369.

[15] Oldham, K. B., Myland, J., and Spanier, J. (2009). An Atlas of Functions with Equator, the Atlas Function Calculator. Springer, New York, USA.

[16] Pearson, K. (1895). Contributions to the mathematical theory of evolution, II: Skew variation in homogeneous material. Philosophical Transactions of the Royal Society of London, A186, 343-414. http://dx.doi.org/10.1098/rsta.1895.0010.

[17] Pearson, K. (1901). Mathematical contributions to the theory of evolution X: Supplement to a memoir on skew of variation. Philosophical Transactions of the Royal Society of London. Series A, Containing Papers of a Mathematical or Physical Character, 197, 343-414. http://dx.doi.org/10.1098/rsta.1901.0023.

[18] Pearson, K. (1916). Mathematical contributions to the theory of evolution, XIX: Second supplement to a memoir on skew of variation. Philosophical Transactions of the Royal Society of London. Series A, Containing Papers of a Mathematical or Physical Character, 216, 429457. http://dx.doi.org/10.1098/rsta.1916.0009.

[19] Prudnikov, A. P., Brychkov, Y. A., and Marichev, O. I. (1986). Integrals and Series, Volume 1, Gordon and Breach Science Publishers, Amsterdam.

[20] Rossani, A., and Scarfone, A. M. (2009). Generalized Pearson distributions for charged particles interacting with an electric and/or a magnetic field. Physica, A, 388, 2354-2366. http://dx.doi.org/10.1016/j.physa.2009.03.001.

[21] Roy, L. K. (1971). An extension of the Pearson system of frequency curves. Trabajos de estadistica y de investigacion operativa, 22 (1-2), 113-123. http://dx.doi.org/10.1007/BF03005269.

[22] Sankaran, P.G. (2003). A generalized Pearson system useful in reliability analysis. Statistical papers, 44, 125-130. http://dx.doi.org/10.1007/s00362-002-0138-3.

[23] Shakil, M., and Kibria, B. M. G. (2010). On a family of life distributions based on generalized Pearson differential equation with applications in health statistics. Journal of Statistical Theory and Applications, 9(2), 255-282.

[24] Shakil, M., Singh, J. N., and Kibria, B. M. G. (2010a). On a family of product distributions based on the Whittaker functions and generalized Pearson differential equation. Pakistan Journal of Statistics, 26(1), 111-125.

[25] Shakil, M., Kibria, B. M. G., and Singh, J. N. (2010b). A new family of distributions based on the generalized Pearson differential equation with some applications. Austrian Journal of Statistics, 39 (3), 259-278.

[26] Shannon, C. E. (1948). A mathematical theory of communication. Bell Systems Technical Journal, 27, pp. 379-423 and pp. 623-656. http://dx.doi.org/10.1002/j.1538-7305.1948.tb01338.x.

[27] Stavroyiannis, S. (2014). On the generalised Pearson distribution for application in financial time series modelling. Global Business and Economics Review, 16(1), 1-14.

[28] Stavroyiannis, S., and Stavroulakis, D. (2007). On the superstatistics with non-linear forces and various distributions. Review of Economic Sciences, 12, 21-34. 